\title{
EXPERIMENTAL TEST OF A NEW ANTIPROTON ACCELERATION SCHEME IN THE FERMILAB MAIN INJECTOR*
}

\author{
V. Wu, C. M. Bhat, B. E. Chase, J. E. Dey, K. G. Meisner, \\ FNAL, Batavia, IL 60510, U.S.A.
}

\begin{abstract}
In an effort to provide higher intensity and lower emittance antiproton beam to the Tevatron collider for high luminosity operation, a new Main Injector (MI) antiproton acceleration scheme has been developed [1-4]. In this scheme, beam is accelerated from 8 to $27 \mathrm{GeV}$ using the $2.5 \mathrm{MHz}$ rf system and from 27 to $150 \mathrm{GeV}$ using the $53 \mathrm{MHz}$ rf system. This paper reports the experimental results of beam study. Simulation results are reported in a different PAC'05 paper [5]. Experiments are conducted with proton beam from the Booster. Acceleration efficiency, emittance growth and beam harmonic transfer between $2.5 \mathrm{MHz}(\mathrm{h}=28)$ and $53 \mathrm{MHz}$ $(\mathrm{h}=588)$ buckets have been studied. Beam study shows that one can achieve an overall acceleration efficiency of about $100 \%$, longitudinal emittance growth less than $20 \%$ and negligible transverse emittance growth.
\end{abstract}

\section{INTRODUCTION}

The Fermilab Tevatron collider is a proton and antiproton facility dedicated for high energy physics research. In order to maximize the integrated luminosity delivered to the collider experiments, the beam intensity and the emittances through the accelerator chain need to be preserved as much as possible. The Main Injector, a $150 \mathrm{GeV}$ synchrotron, serves as the injector to the Tevatron. Currently the antiproton bunches for Tevatron are produced by a multi-bunch coalescing scheme in the MI at $150 \mathrm{GeV}$ [6]. This method yields about a factor of two increase in the longitudinal emittance and 5\% to $20 \%$ decrease in intensity before injection to Tevatron. Most of the emittance growth and beam loss comes from the coalescing process. In an effort to improve emittance growth and beam loss, a new antiproton acceleration scheme, called $2.5 \mathrm{MHz}$ acceleration for short, is developed. According to simulations [5], the new method can potentially reduce the emittance growth to the $10 \%$ level without beam loss.

The $2.5 \mathrm{MHz}$ acceleration method uses both the Main Injector $2.5 \mathrm{MHz}$ and $53 \mathrm{MHz}$ rf systems for beam acceleration. Figure 1 shows a schematic view of the principle behind this method. At present, all of the antiproton injections to the MI are in $2.5 \mathrm{MHz}$ buckets [7]. Therefore, it is natural to begin MI acceleration in $2.5 \mathrm{MHz}$ buckets. Four $2.5 \mathrm{MHz}$ bunches are accelerated through transition (about $20.4 \mathrm{GeV}$ ) from 8 to $27 \mathrm{GeV}$. At the constant energy $27 \mathrm{GeV}$, the bunches are transferred

\footnotetext{
* Operated by Universities Research Association, Inc. for the U.S. Department of Energy under contract DE-AC02-76CH03000.

\#vincentw@fnal.gov
}

to $53 \mathrm{MHz}$ buckets after two rotations. Then the beam is accelerated to $150 \mathrm{GeV}$ and injected to the Tevatron. The multi-bunch coalescing process is eliminated in this acceleration scheme. Consequently, longitudinal emittance growth is reduced. Smaller emittance growth reduces beam loss.

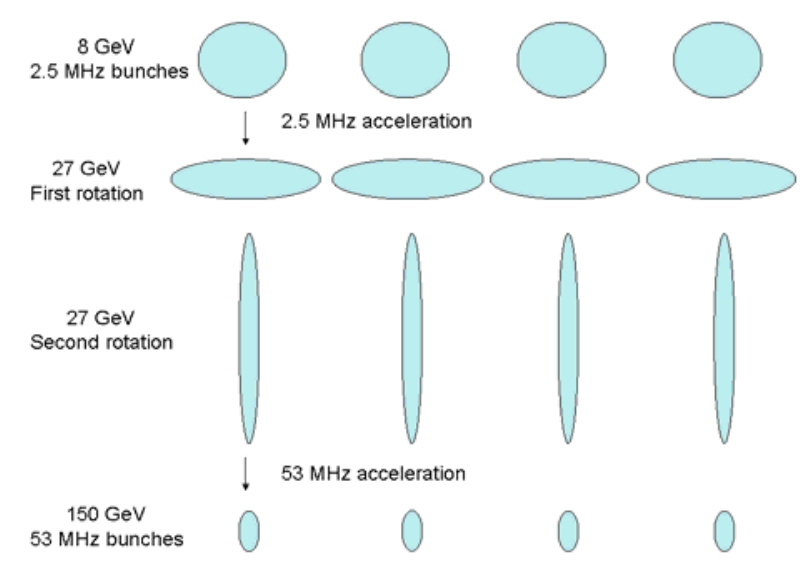

Figure 1: The $2.5 \mathrm{MHz}$ acceleration scheme. The acceleration begins with four consecutive $2.5 \mathrm{MHz}$ bunches. At $27 \mathrm{GeV}$, the bunches undergo two one quarter synchrotron period rotations. When the bunches are at minimum bunch widths at the end of the second rotation, they are transferred to matched $53 \mathrm{MHz}$ buckets. Then the $53 \mathrm{MHz}$ bunches are accelerated to $150 \mathrm{GeV}$ and injected into the Tevatron.

\section{EXPERIMENTS}

Proton beam is used to test the feasibility of the $2.5 \mathrm{MHz}$ acceleration scheme. The $2.5 \mathrm{MHz}$ bunches are prepared in the MI at $8 \mathrm{GeV}$ by injecting four short batches of $53 \mathrm{MHz}$ bunches from the Fermilab Booster; see Fig. 2 for mountain range picture. These batches are spaced by twenty-one $53 \mathrm{MHz}$ buckets (398 nsec apart) for creating four consecutive $2.5 \mathrm{MHz}$ bunches. The numbers of Booster turn and $53 \mathrm{MHz}$ bunches in each batch depend on the desired initial $2.5 \mathrm{MHz}$ intensity and longitudinal emittance. After injection, the $53 \mathrm{MHz}$ rf voltage is adiabatically reduced to debunch the $53 \mathrm{MHz}$ bunches inside $2.5 \mathrm{MHz}$ buckets. At the end of debunching, the high power inputs to the $53 \mathrm{MHz}$ cavities are switched off. The measured total residual rf voltage in the $53 \mathrm{MHz}$ cavities (due to bleed through problem) is of the order of $100 \mathrm{~V}$ [8]. Both $2.5 \mathrm{MHz}$ and $53 \mathrm{MHz}$ beam loading compensations are on during beam studies. 


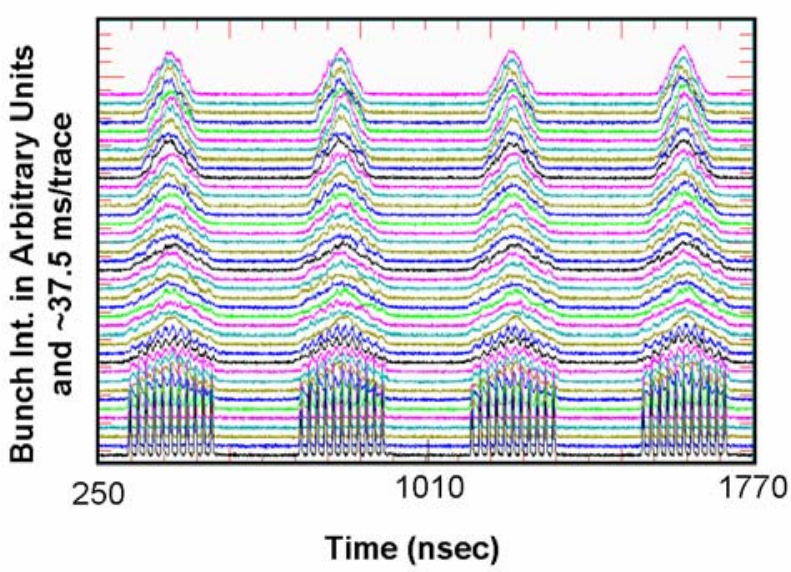

Figure 2: (Color) Mountain range picture showing the process of creating four $2.5 \mathrm{MHz}$ bunches in the Main Injector at $8 \mathrm{GeV}$. In this case, 4 batches of 11 Booster proton bunches $(53 \mathrm{MHz})$ are injected into the Main Injector. The $53 \mathrm{MHz}$ bunches are adiabatically debunched inside $2.5 \mathrm{MHz}$ buckets. Then the $2.5 \mathrm{MHz}$ bunches are shrunk adiabatically prior to acceleration.

Once the $2.5 \mathrm{MHz}$ bunches are prepared, they are accelerated from 8 to $27 \mathrm{GeV}$ with the $2.5 \mathrm{MHz}$ rf system and from 27 to $150 \mathrm{GeV}$ with the $53 \mathrm{MHz}$ rf system. Acceleration is performed with closed phase feed-forward and radial position feedback loops. Since the acceleration involves two different frequencies, two sets of phase and radial position detectors and feedback loops are used. At the $27 \mathrm{GeV}$ constant energy, the $2.5 \mathrm{MHz}$ bunches undergo two one quarter synchrotron period rotations. The first rotation is at about $4 \mathrm{kV}$ for reducing the bunch height to an acceptable level (a few $\mathrm{MeV}$ ). The second rotation is a fast rotation at $60 \mathrm{kV}$. When the bunches are at minimum bunch widths (less than 18.9 nsec, the width of $53 \mathrm{MHz}$ bucket), $53 \mathrm{MHz}$ cavities are switched back on and capture the bunches with matched buckets. Figure 3 shows mountain range picture of the rotations and the beam harmonic transfers to $53 \mathrm{MHz}$ buckets. After $53 \mathrm{MHz}$ captures, the phase and the radial position loops switch from $2.5 \mathrm{MHz}$ to $53 \mathrm{MHz}$ before beam acceleration to $150 \mathrm{GeV}$.

Figure 4 shows the $150 \mathrm{GeV}$ momentum ramp, the $2.5 \mathrm{MHz}$ and the $53 \mathrm{MHz}$ rf amplitude curves and the beam intensity for the case of initial bunch intensity $60 \times 10^{9}$ and bunch emittance 2 eVs. The slow acceleration (2.5 MHz) portion of the ramp has a maximum $\mathrm{dP} / \mathrm{dt}$ of $3.2(\mathrm{GeV} / \mathrm{c}) / \mathrm{sec}$. (At the moment, the maximum $\mathrm{dP} / \mathrm{dt}$ can be used for $2.5 \mathrm{MHz}$ acceleration is limited by maximum available $2.5 \mathrm{MHz}$ rf voltage of $75 \mathrm{kV}$.) For the fast acceleration (53 MHz), the maximum acceleration rate is $120(\mathrm{GeV} / \mathrm{c}) / \mathrm{sec}$. The acceleration efficiency of the beam shown here from 8 to $27 \mathrm{GeV}$ is $100 \%$ and from 27 to $150 \mathrm{GeV}$ is about $98 \%$. Overall, the $53 \mathrm{MHz}$ capture efficiency is in the range of $90 \%$ to $100 \%$.

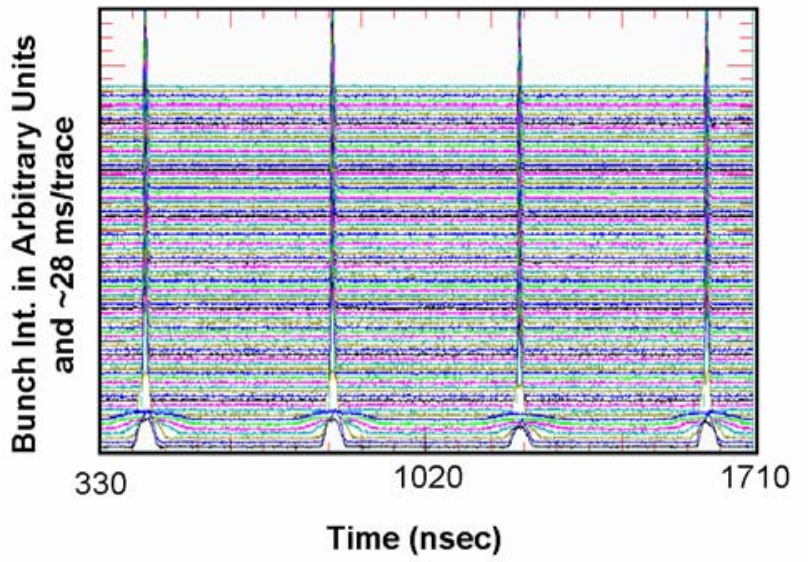

Figure 3: (Color) Mountain range picture showing the rotations and the beam harmonic transfer to $53 \mathrm{MHz}$ buckets at $27 \mathrm{GeV}$. After $53 \mathrm{MHz}$ captures, the bunches are accelerated to $150 \mathrm{GeV}$.

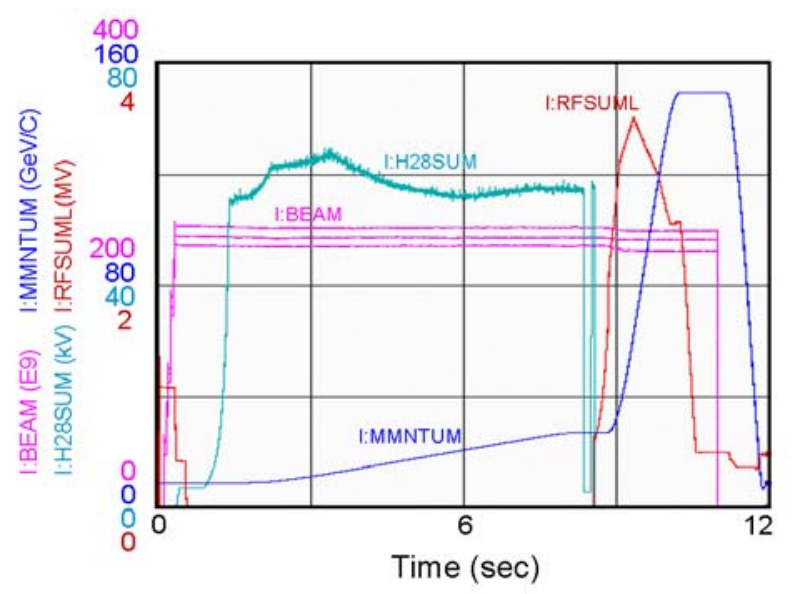

Figure 4: (Color) The $150 \mathrm{GeV}$ momentum ramp (I:MMNTUM, blue), $2.5 \mathrm{MHz}$ (I:H28SUM, green) and $53 \mathrm{MHz}$ (I:RFSUM, red) rf curves and the beam intensity (I:BEAM, $10^{9}$, pink) vs. cycle time (sec) for four batch injections. The total intensity is about $24 \times 10^{10}$. The acceleration efficiency is about $98 \%$.

The longitudinal emittance [9] of the beam at injection and at flat-top $(150 \mathrm{GeV})$ are determined by measuring the bunch lengths and the rf voltages. The bunch emittance is calculated when the beam is in matched stationary bucket. Table 1 lists the measured longitudinal emittance for two cases of initial emittance and intensity. The 95\% longitudinal emittance is calculated using the bunch length that corresponds to $95 \%$ of the area (total bunch intensity) under the Wall Current Monitor bunch signal. For the measurements, the operational parameters, viz., rotation voltages and time, $53 \mathrm{MHz}$ capture time, $2.5 \mathrm{MHz}$ alignment, etc., are tuned up for the case of $2 \mathrm{eVs}$ initial emittance. Thus, the case of $0.9 \mathrm{eVs}$ initial emittance is not optimized and has more emittance growth. For the optimized case, the amount of longitudinal emittance growth and the acceleration efficiency are consistent with the simulations. 
Table 1: Longitudinal emittance (95\%) measurements at injection and at $150 \mathrm{GeV}$ flat-top for four batches injection. Measurement error is 10 to 20 percents.

\begin{tabular}{|l|l|l|}
\hline Total Intensity (10 $\mathbf{9})$ & 130 & 240 \\
\hline Initial 8 GeV Emittance (eVs)/bunch & 0.9 & 2.0 \\
\hline 150 GeV Emittance (eVs)/bunch & 1.5 & 2.2 \\
\hline Emittance Growth (\%) & 61 & 13 \\
\hline Overall Beam Loss (\%) & 1 & 8 \\
\hline
\end{tabular}

The longitudinal emittance growth comes from two places, namely, transition crossing and rf manipulations at $27 \mathrm{GeV}$. Because of the relatively long transition region (about $150 \mathrm{~ms}$ for the non-adiabatic and non-linear regions), there is always a certain amount of beam mismatch to bucket after transition crossing. Bunch shape oscillation is observed after transition crossing (see Fig. 5) that leads to emittance growth. At $27 \mathrm{GeV}$, imperfect tuning in rotation voltages and timings, $2.5 \mathrm{MHz}$ alignment, $53 \mathrm{MHz}$ capture voltage, beam loading compensations, etc., can lead to emittance growth.

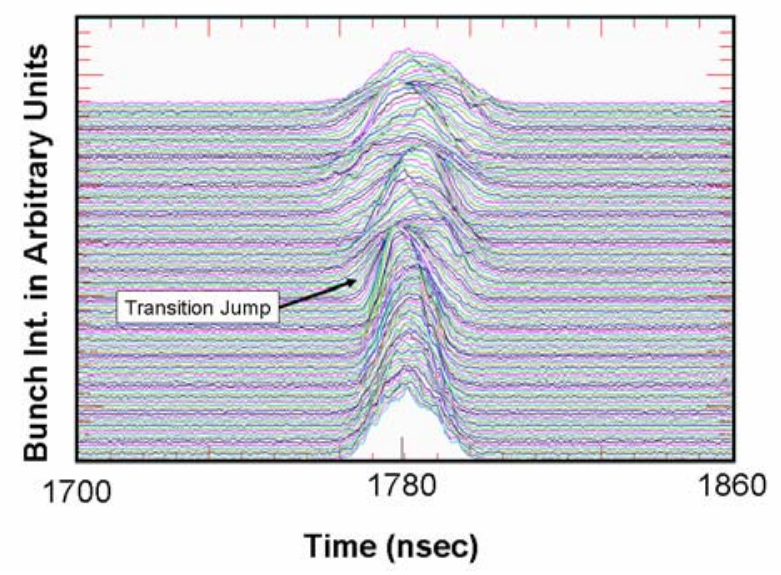

Figure 5: (Color) Mountain range picture showing transition crossing. Bunch shape oscillation is observed after transition jump which leads to emittance growth.

The transverse emittances of the beam are measured using the flying wires at various times up the ramp. Figure 6 shows typical flying wire data taken at 8 and $150 \mathrm{GeV}$. The measured transverse emittances are $8.9 \pi \cdot \mathrm{mm} \cdot \mathrm{mr}$ (vertical) and $9 \pi \cdot \mathrm{mm} \cdot \mathrm{mr}$ (horizontal) at $8 \mathrm{GeV}$ and are $9.8 \pi \cdot \mathrm{mm} \cdot \mathrm{mr}$ (vertical) and $10.1 \pi \cdot \mathrm{mm} \cdot \mathrm{mr}$ (horizontal) at $150 \mathrm{GeV}$ with about $10 \%$ measurement error. Thus, the transverse emittance growth is negligible.

\section{CONCLUSIONS}

A new antiproton acceleration scheme for producing high intensity and low emittance bunches for the Tevatron collider is presented. Experimental test of the acceleration scheme is carried out in the Main Injector using proton beam from the Booster. Beam study results are very promising. The longitudinal emittance growth is less than
$20 \%$ and there is negligible transverse emittance growth. The overall acceleration efficiency is about 100 percents.

In the future, we plan to implement this scheme for antiproton acceleration in the MI for the Tevatron shots and expect about 5-10\% higher instantaneous protonantiproton luminosity at the collider detectors.

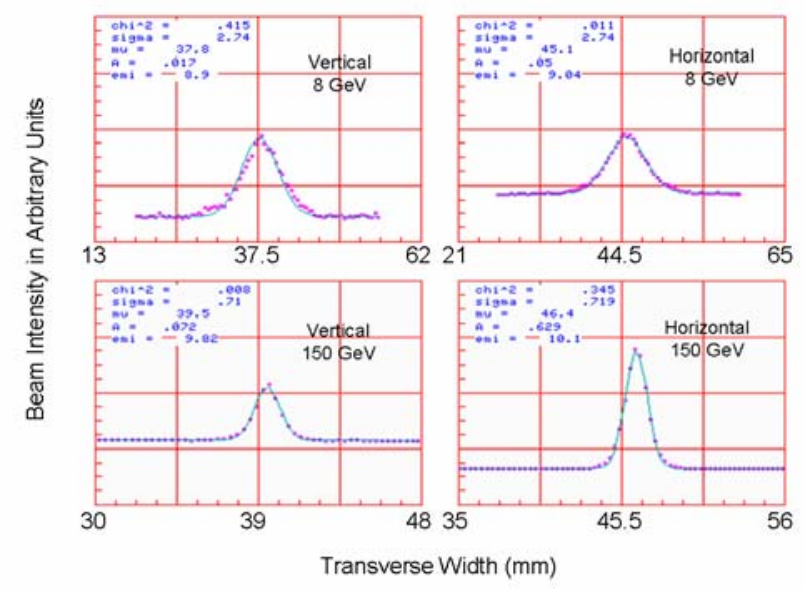

Figure 6: (Color) Transverse emittance measurements at 8 and $150 \mathrm{GeV}$ using flying wires. The four plots show the vertical beam profiles (on the left) and the horizontal beam profiles (on the right). The overall transverse emittance growths in both planes are about $1 \pi \cdot \mathrm{mm} \cdot \mathrm{mr}$.

\section{REFERENCES}

[1] G. P. Jackson, FERMILAB-TM-1991, November 1996.

[2] I. Kourbanis and D. W. Wildman, PAC1999, page 2840, 1999.

[3] J. A. MacLachlan, FERMILAB-TM-2177, July 2002.

[4] C. M. Bhat and J. E. Dey, "Pbar Acceleration in MI using 2.5 MHz Coalescing RF system - Tune-up Study Scheme with Proton Beam and Operational Implementation”, MI Note 272, February 2001.

[5] V. Wu, et al,"Simulations of an Acceleration Scheme for Producing High Intensity and Low Emittance Antiproton Beam for Fermilab Collider Operation”, this conference (PAC'05).

[6] J. E. Griffin et al, IEEE, Trans. Nucl. Sci., NS-30, No. 4, 1983.

[7] C. M. Bhat et al.,"2.5 MHz Pbar Bunch Transfer from Accumulator and Recycler to the Fermilab Main Injector and Pbars to the Tevatron PPbar Collider", this conference.

[8] J. Dey and I. Kourbanis, “53 MHz Beam Loading Compensation for Slip Stacking in the Fermilab Main Injector”, this conference (PAC'05).

[9] P. S. Martin and S. Ohnuma, AIP Proceedings, no. 184, 1989. 